\title{
High-resolution imaging of elastic properties using harmonic cantilevers
}

\author{
O. Sahin ${ }^{\mathrm{a}, *}$, G. Yaralioglu ${ }^{\mathrm{a}}$, R. Grow ${ }^{\mathrm{a}}$, S.F. Zappe ${ }^{\mathrm{a}}$, A. Atalar ${ }^{\mathrm{b}}$, C. Quate $^{\mathrm{a}}$, O. Solgaard ${ }^{\mathrm{a}}$ \\ ${ }^{a}$ E.L. Ginzton Laboratory, Stanford University, Stanford, CA 94305-4085, USA \\ ${ }^{\mathrm{b}}$ Department of Electrical and Electronics Engineering, Bilkent University, Ankara 06533, Turkey
}

Received 1 July 2003; received in revised form 12 November 2003; accepted 21 November 2003

Available online 21 February 2004

\begin{abstract}
We present a micromachined scanning probe cantilever, in which a specific higher-order flexural mode is designed to be resonant at an exact integer multiple of the fundamental resonance frequency. We have fabricated such cantilevers by reducing the stiffness of the third order flexural mode relative to the fundamental mode, and we have demonstrated that these cantilevers enable sensing of non-linear mechanical interactions between the atomically sharp tip at the free end of the cantilever and a surface with unknown mechanical properties in tapping-mode atomic force microscopy. Images of surfaces with large topographical variations show that for such samples harmonic imaging has better resolution than standard tapping-mode imaging.
\end{abstract}

(C) 2003 Elsevier B.V. All rights reserved.

Keywords: Atomic force microscope; Tapping-mode; Cantilever; Elastic properties

\section{Introduction}

Atomic force microscope (AFM) [1] has proven to be an exceptionally useful tool for mapping topography of surfaces at the nanoscale. In an AFM, a flexible cantilever with an atomically sharp tip is brought to the vicinity of a sample surface and the deflections of the cantilever as a result of the attractive and repulsive forces between the tip and the sample are monitored while the cantilever is scanned across the surface.

The earliest AFM imaging techniques required continuous contact between the tip and sample, which resulted in frictional forces that damage both the tip and the sample. To overcome this problem, dynamic imaging techniques that do not require continuous tip-sample contact have been introduced. Among these, tapping-mode atomic force microscope (TM-AFM) [2] has become the most widely used. In TM-AFM (Fig. 1), the cantilever is vibrated at a frequency close to one of its flexural resonances in vicinity of the surface under test so that the tip makes intermittent contacts (tapping) with the surface once in every oscillation period. During the scan across the surface, the amplitude of vibration is maintained at a constant value through a feedback

\footnotetext{
* Corresponding author. Tel.: +1-650-4980778; fax: +1-650-7252533. E-mail address: sahin@stanford.edu (O. Sahin).
}

loop that adjusts the height of the cantilever base. The feedback signal therefore reflects the topography of the surface.

Apart from high-resolution topography imaging, there has been a great deal of interest in using AFM as a tool to probe the hardness and elasticity at the nanoscale. Imaging of elastic properties enables detection of variations in chemical composition across the surface, characterization of thin films, and studies of nanomechanical structures in general. With an AFM one can measure the contact stiffness between the nano-tip and the sample, which is a measure of the elasticity of the material in the vicinity of the contact. Various techniques for measuring contact stiffness has been developed, including quasi-static ones that apply force to the sample and measure the indentation of the surface simultaneously $[3,4]$, and dynamic ones that either vibrate the cantilever or the sample and measure variations in the response of the cantilever which is affected by the elasticity of the sample [5-9]. These techniques require continuous tip-sample contact and therefore, they pose the same problems as contact-mode imaging. Such techniques are not suitable for imaging soft samples like biological samples and polymers. The techniques that have been demonstrated also have not achieved sufficient sensitivity and simplicity for practical imaging.

Studies of cantilever dynamics in TM-AFM have shown that the vibration spectrum of tapping cantilevers exhibit peaks at integer multiples (higher harmonics) of the excita- 


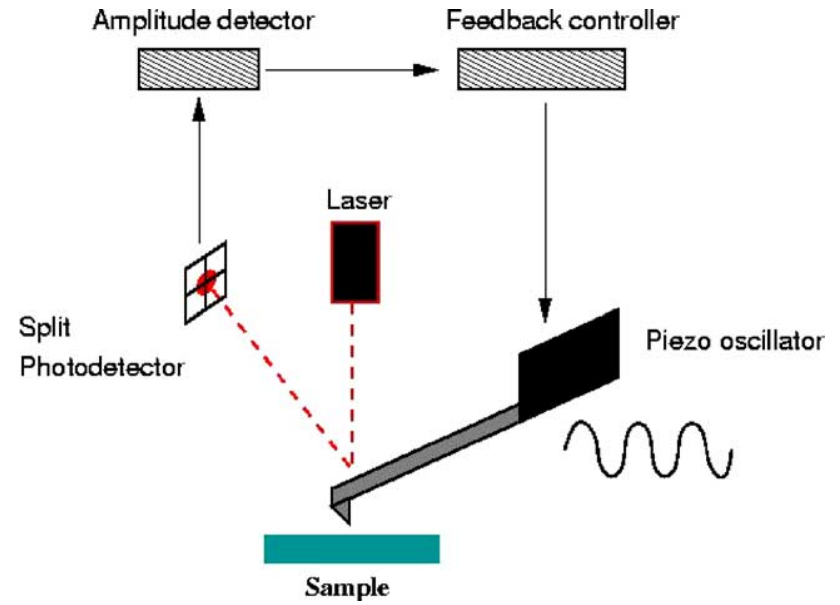

Fig. 1. Schematic of tapping-mode operation. A cantilever with a tip is vibrated with a piezo-element and its deflection amplitude is monitored and kept constant with an optical lever detection system and a feedback controller.

tion frequency [10]. These harmonics are generated by the non-linearity of the tip-sample interaction and carry information about the mechanical properties of the sample on a nanometer spatial scale [11]. Imaging with these higher harmonics has been demonstrated and the result has shown good contrast based on material properties [12]. Unfortunately, the vibration amplitudes of traditional AFM cantilevers at higher harmonics are too small for practical AFM imaging [13]. Here we report a new micromachined cantilever, the harmonic cantilever, which will enable practical and non-destructive measurement of elasticity on both soft and hard samples with a good accuracy by enhancing the non-linear energy transfer to higher harmonics in TM-AFM.

\section{Theory}

In TM-AFM the cantilever is vibrated at its fundamental resonance frequency with sufficiently large amplitudes $(10-100 \mathrm{~nm})$ to avoid sticking. The distance between the sample surface and the rest position of the tip is kept shorter than the free vibration amplitude so that tip hits the surface once every period. Therefore, the amplitude of the cantilever vibration is reduced to the tip-sample separation. The duration of the intermittent contacts can be as long as $15 \%$ of the oscillation period, depending on the hardness of the sample [14]. On softer samples the tip can indent the sample surface more and spend more time in contact. Other than the repulsive forces that appear when the tip indents the surface, there are also attractive forces between the tip and the sample. The origins of these attractive forces are mainly capillary forces and Van der Waals forces. They appear when the tip gets closer to the surface. An illustration of the interaction forces and tip displacement is given in Fig. 2. In general, the attractive forces are higher when the tip retracts. This is either due to a hysteresis in Van der Waals forces or due to formation and disruption of a liquid neck.

The periodic nature of tip-sample forces results in higher-order Fourier components (harmonics) exactly at integer multiples of the vibration frequency of the cantilever.

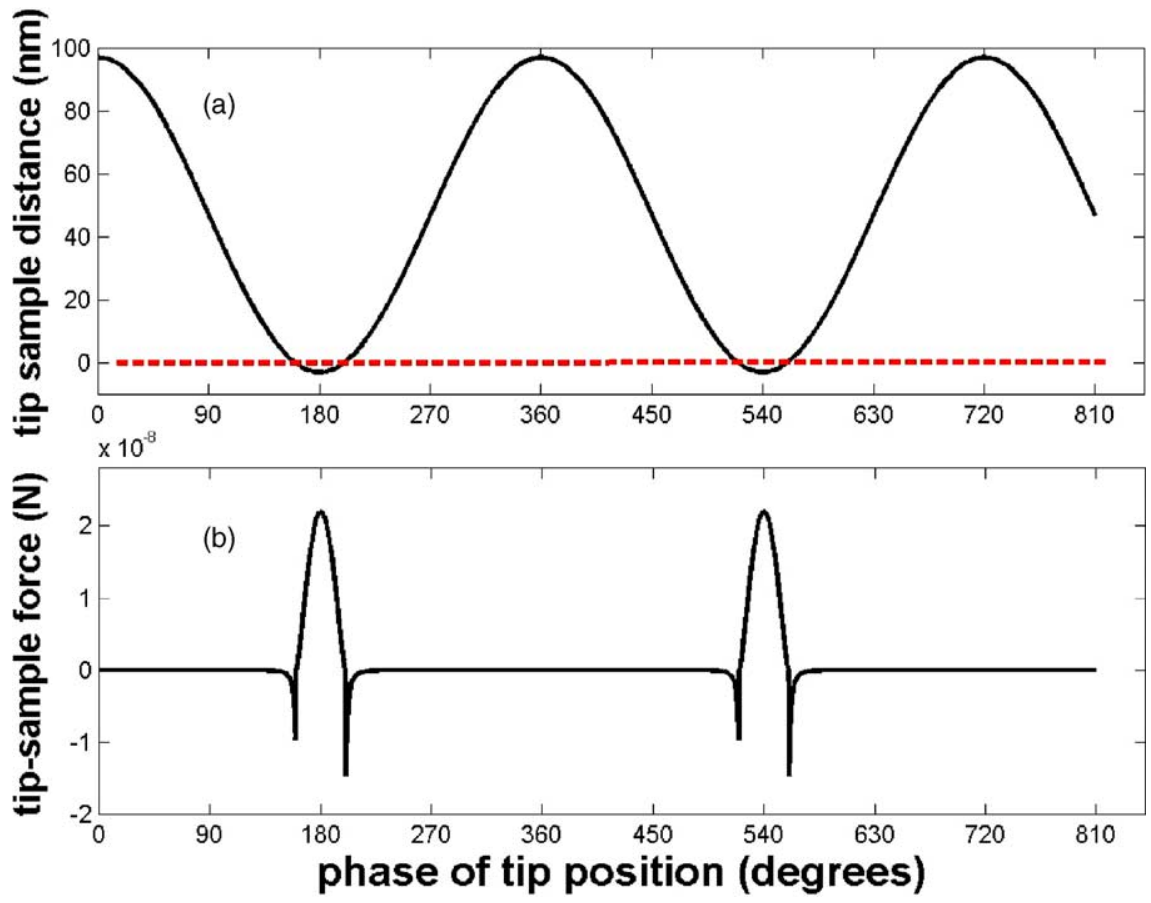

Fig. 2. Tip position (a) with respect to sample surface (dashed line) in TM-AFM and tip-sample interaction forces (b). Negative tip displacement means that tip indents the sample surface. 

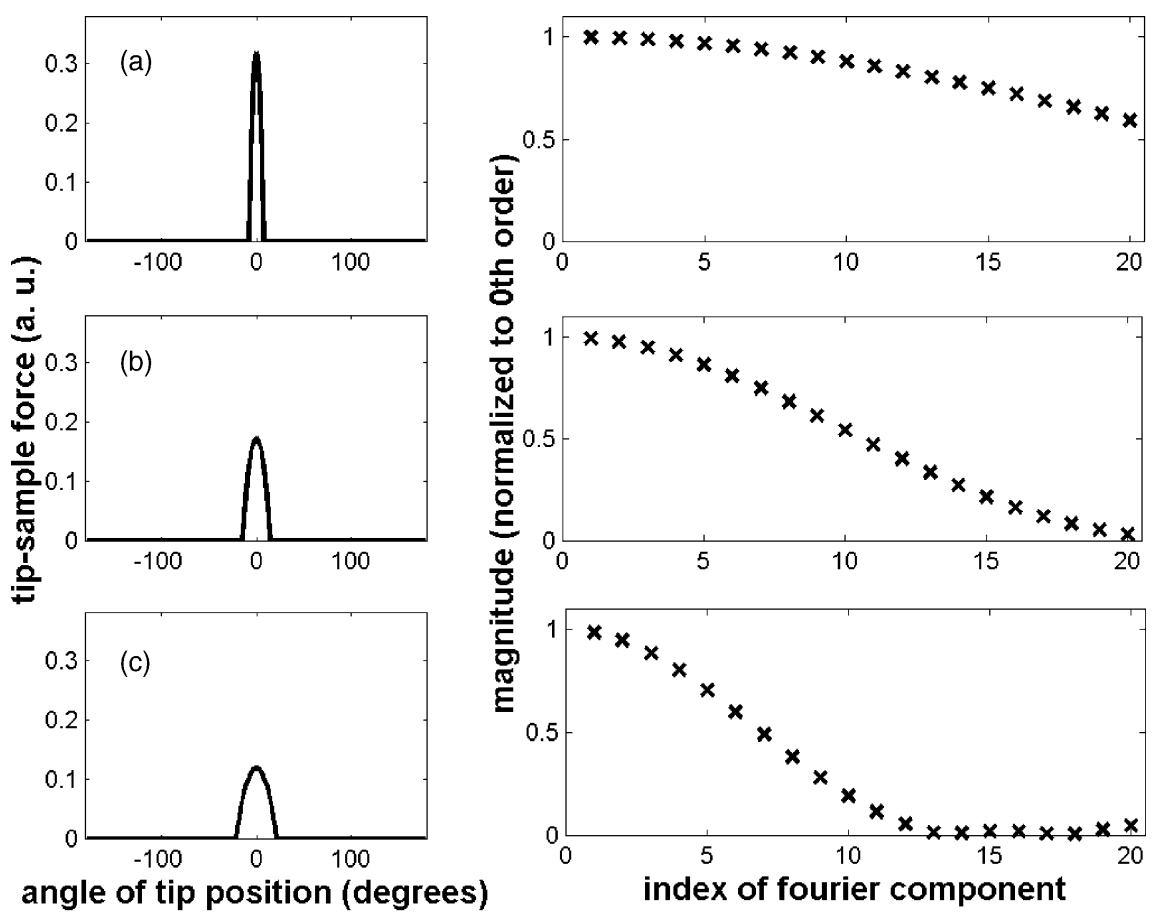

Fig. 3. Tip-sample interaction forces and corresponding Fourier spectrum of forces for (a) $4 \%$; (b) $8 \%$ and (c) $12 \%$, contact times. The average forces are normalized to 1 in all three cases.

The magnitudes of these harmonics mainly depend on the contact time and peak force. For illustrative purposes we modeled the sample with a linear spring and neglected the attractive forces. Under these circumstances, the interaction forces become a clipped sine wave. In Fig. 3, we plot the magnitudes of the harmonic forces calculated with this model for three different contact times, 4,8 and $12 \%$ corresponding to hard medium and soft samples. In order to compare the three different cases, we assumed that the average force (0th order Fourier component) is the same in all of them $[15,16]$. Then we normalized the magnitudes of the harmonics to the 0th order Fourier component. These plots show that the higher-order components, especially the ones around 15th harmonic in our example, depend highly on the contact time. Therefore, one can argue that in TM-AFM the information about the sample elasticity

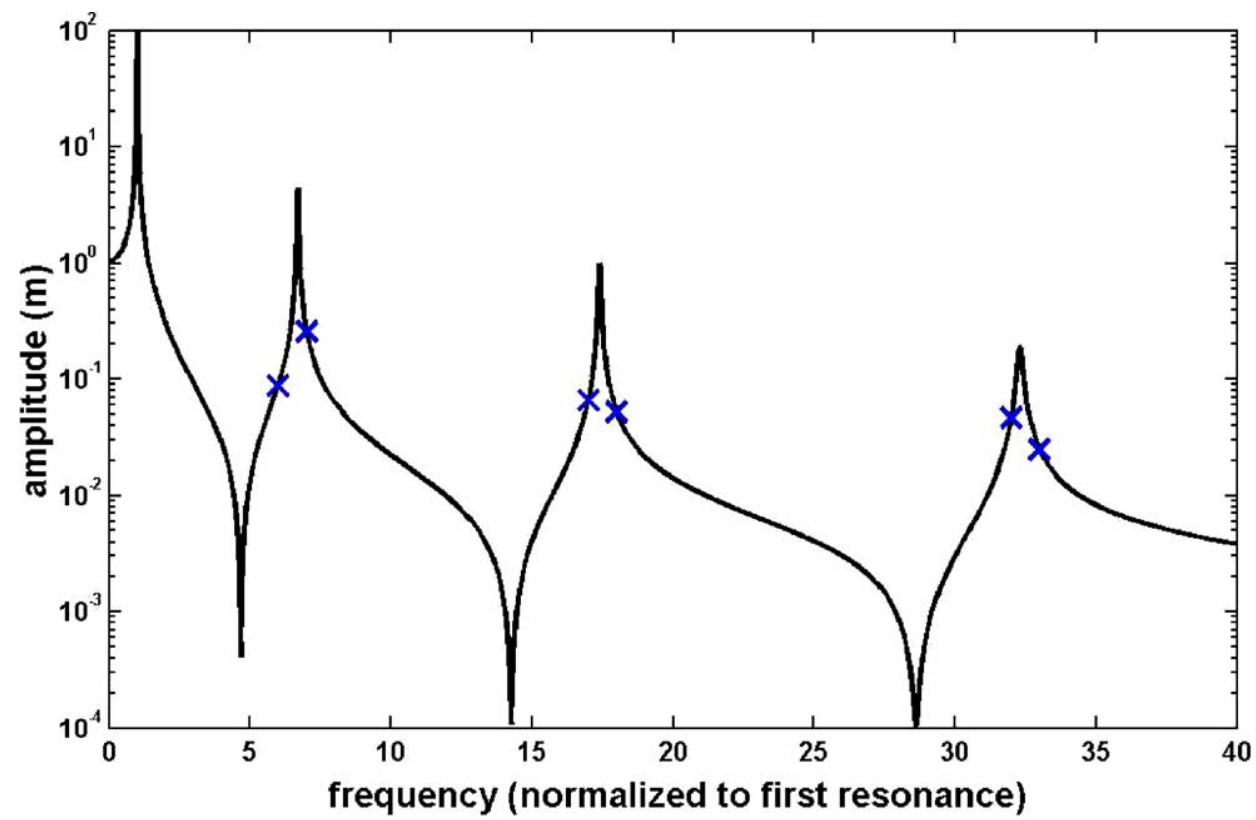

Fig. 4. Transfer function of a typical AFM cantilever. The cross marks point the location of the harmonic forces that are closest to the resonance frequencies. 
is in the higher harmonics of the tip-sample interaction forces.

The higher harmonics are all driven by the forces acting on the cantilever tip. The actual cantilever displacements at these frequencies therefore depend on the transfer function of the cantilever relating displacement to the tip force. That is, the higher harmonic forces, which have frequencies close to resonances of the cantilever, will generate larger vibration amplitudes. Fig. 4 gives the transfer function of a rectangular cantilever whose fundamental resonance frequency is normalized to 1 . The higher harmonics that are closest to the resonance peaks are also marked on the curve. Typical quality factors of each resonance are on the order of several hundreds. For a randomly picked cantilever it is therefore very unlikely that one of the higher harmonics coincide with one of the resonances of the cantilever. As a result of this, none of the higher harmonics of a typical cantilever makes use of the high transfer function at the resonance peaks.

\section{Harmonic cantilever}

We have designed and fabricated an AFM cantilever with increased vibration amplitude at higher harmonics by matching a higher-order natural resonance to an integer multiple of the fundamental resonance frequency. Specifically, we matched the third order flexural resonance frequency to the 16 th harmonic of the fundamental. Note that the 16th harmonic is very sensitive to contact time variations (see Fig. 3).

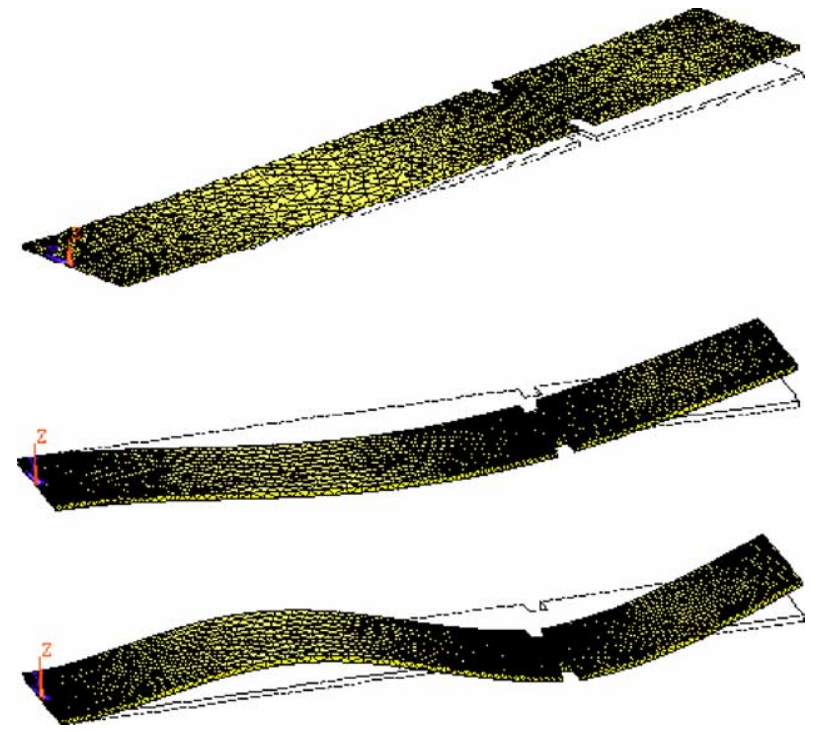

Fig. 5. Mode shapes of a rectangular notched cantilever fixed at one end and free at the other end. The notch is located at the highly curved region of third mode, approximately $1 / 3$ of the total length away from the free end.

\subsection{Design approach}

Tuning the resonance frequency of the third order mode is achieved by appropriately removing mass from regions where the cantilever has high mechanical stress in that

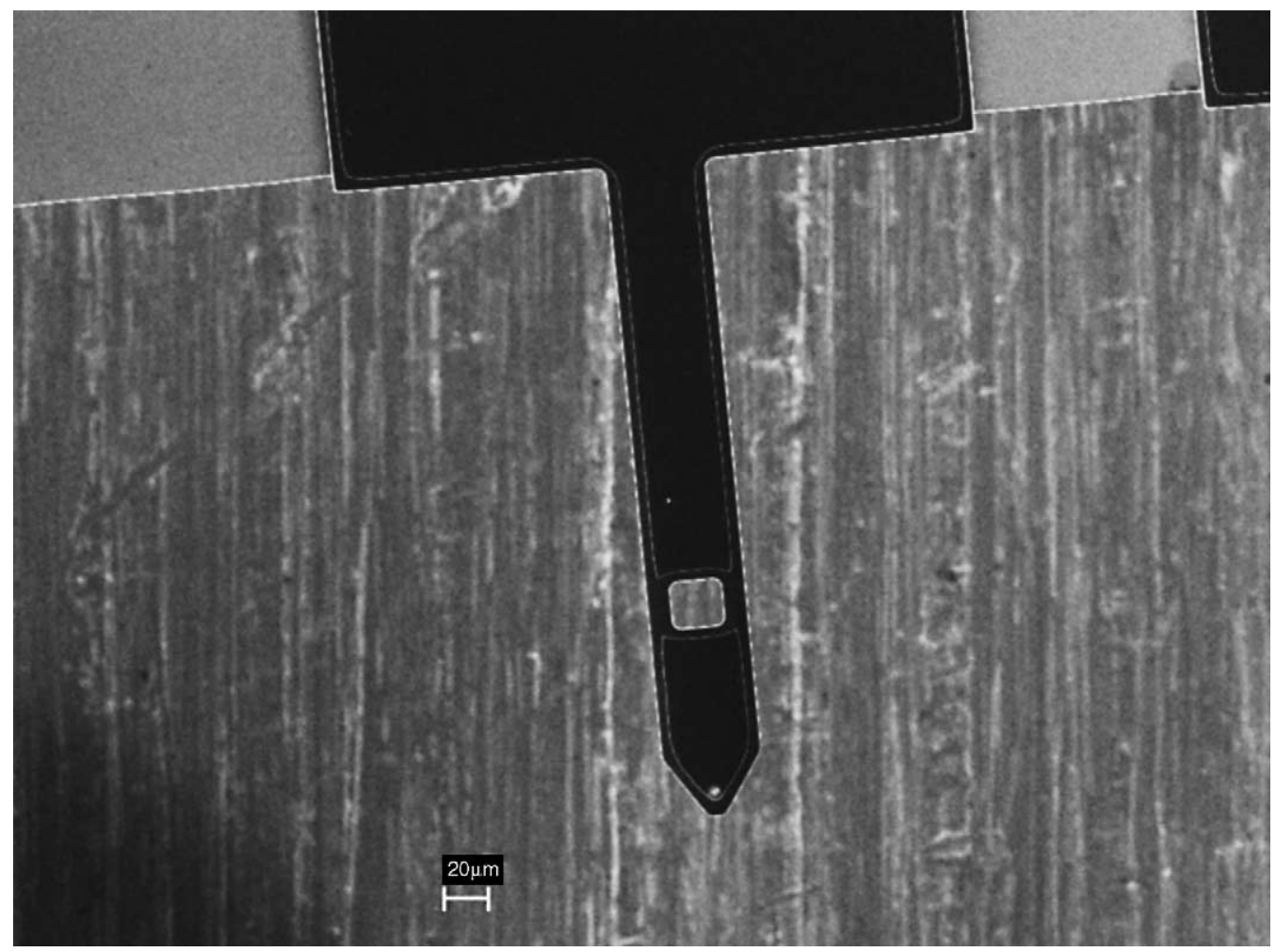

Fig. 6. SEM image of a harmonic cantilever. Width, length and thickness of the cantilever are 50, 300, and $2.2 \mu \mathrm{m}$, respectively. The rectangular opening is $22 \mu \mathrm{m} \times 18 \mu \mathrm{m}$ and centered $190 \mu \mathrm{m}$ away from the cantilever base. 
(a) Top view

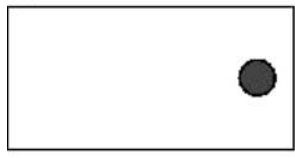

(b) Side view

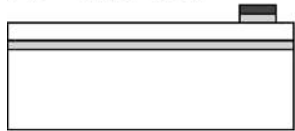

(c) Side view

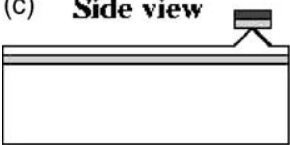

(d) Side view

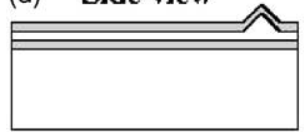

(e) Top view

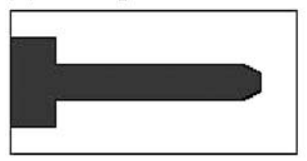

(f) Side view

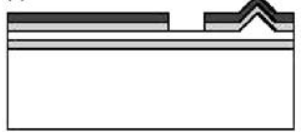

(g) Side view

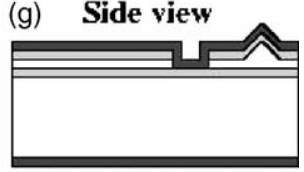

(h) Side view

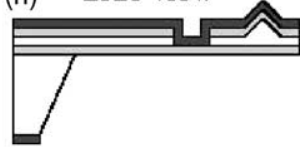

(i) Side view

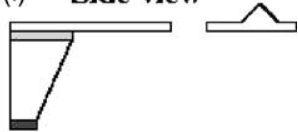

nitride

Fig. 7. Process steps of the fabrication of harmonic cantilevers.

particular mode, thereby reducing elastic energy and the resonance frequency of the mode. To illustrate this, the first three flexural mode shapes of a rectangular cantilever with a notch are given in Fig. 5. The cantilever is fixed at the base and free at the other end. The notch is located at one of the curved regions of the third mode. Note that this location does not correspond to a highly curved region in the first two modes. Therefore, the effect of the notch in reducing the elastic energy is more prominent in the third mode. In addition to the elastic energy, the kinetic energy in a mode also affects the resonant frequency of a mode. These two forms of energy correspond to the effective spring constant (elastic energy) and effective mass (kinetic energy) of a mode. Since removal of mass changes the mass distribution of the cantilever, it also affects the kinetic energies in each mode. Therefore, the effect of variations in the kinetic energy has to

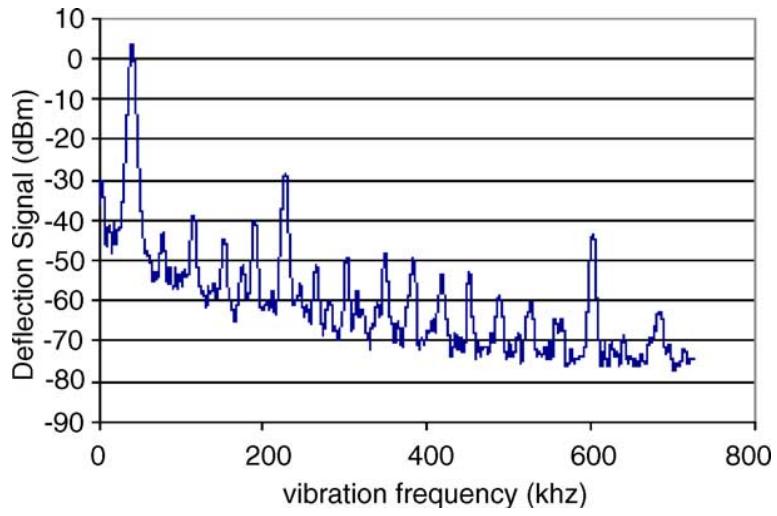

Fig. 8. Vibration spectrum of a harmonic cantilever in tapping-mode AFM. The cantilever is driven at its fundamental resonance frequency $(37.4 \mathrm{kHz})$, and higher-harmonics generation is observed. The second $(240 \mathrm{kHz})$ and third $(598 \mathrm{kHz})$ harmonics coincides with higher resonances and have relatively large signal power.
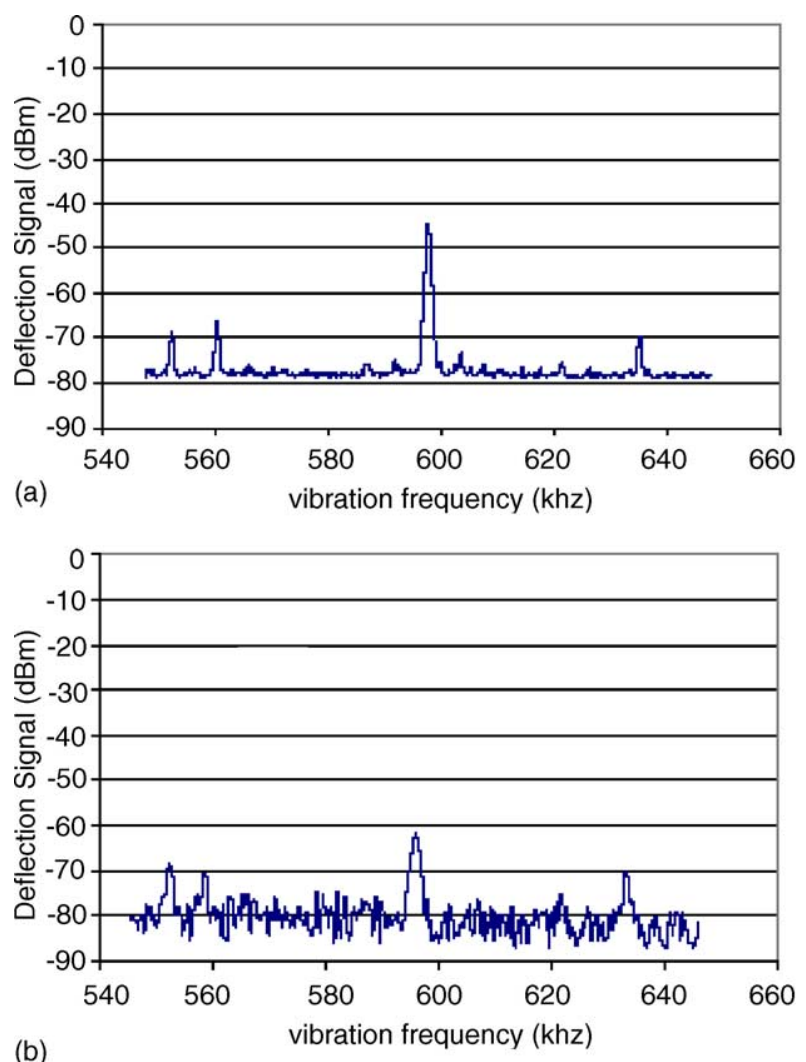

Fig. 9. Spectrum of the vibrations of a harmonic cantilever around 15th-17th harmonics of the driving frequency in tapping-mode atomic force microscopy. (a) The 16th harmonic is exactly equal to the third resonance frequency of the cantilever, recorded at $300 \mathrm{~Hz}$ bandwidth. (b) The 16th harmonic is slightly less than the third resonance frequency $(596 \mathrm{kHz}$ instead of $598 \mathrm{kHz})$, recorded at $1 \mathrm{kHz}$ bandwidth. 
be taken into account while designing a harmonic cantilever. In our case, when the mass is removed from the curved region of the third flexural mode as shown in Fig. 5, variations in the elastic energy is more significant than in kinetic energy. This is because at the location of the notch, both the first and the third modes have similar displacements. However; the curvature in the third mode at that location is much higher than its counterpart in the first mode. Also, the second mode is minimally affected because the location of the notch is close to a node of that mode where the curvature and the displacement are both small. Please refer to Stark and Heckl [17] for analytical expressions on the effective masses and spring constants of the modes of a cantilever.

\subsection{Fabrication}

Instead of tuning the resonances with by creating notches at a given location, we preferred opening holes in the cantilevers at these same locations. This way the cantilevers will be more stable in terms of torsional vibrations. A scanning electron micrograph image of a harmonic cantilever is given in Fig. 6. With finite element analysis, the hole in the cantilever is designed to give the correct value for the resonance frequency of the third order flexural mode. However, a series of cantilevers were fabricated with slightly different hole sizes $(2 \mu \mathrm{m}$ steps in width perpendicular to the length of the beam) to allow for dimensional variation due to the fabrication process.

The fabrication of harmonic cantilevers is a three-mask process. The schematic of the process steps is given in Fig. 7. First, the tip masks (a) are transferred to an oxide/nitride hard mask on the device layer of an SOI wafer (b). Tips are plasma etched (c) and oxide sharpened (d). The second mask defines the cantilever geometry, including the holes (e). The cantilever pattern is transferred to the nitride and oxide layers by plasma etch and buffered oxide etch. Then the cantilevers are formed in a plasma etch (f). The front and back side of the wafer is then covered with nitride $(\mathrm{g})$, and the back side is patterned for $\mathrm{KOH}$ etching (h). After etching through the wafer, the cantilevers are released by removing the protective nitride layer in plasma etch and removing the oxide in a buffered oxide etch (i).

Non-uniform etch rates resulted in variations in cantilever thicknesses, which in turn translated into variations in the
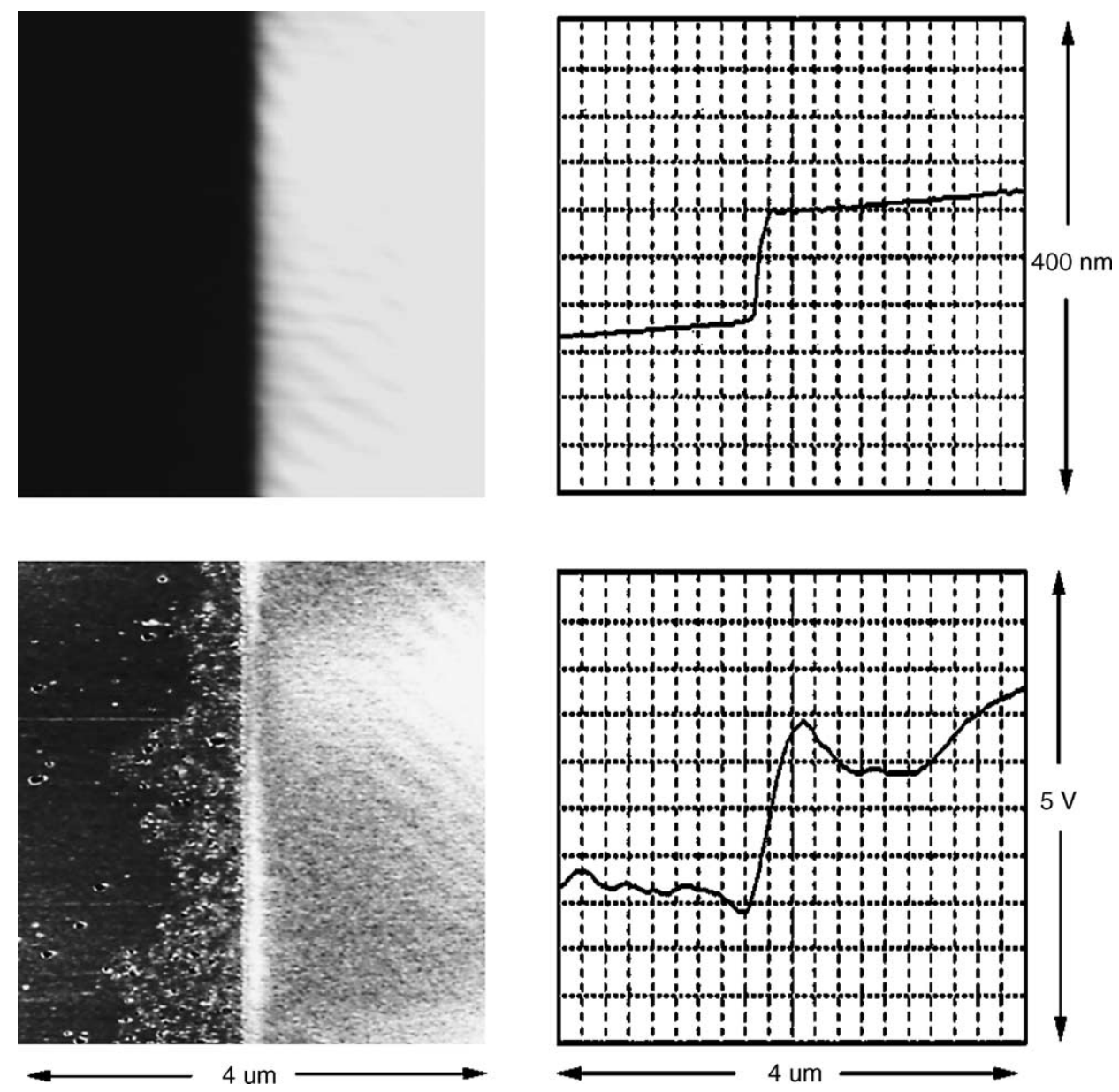

Fig. 10. Images of chrome edge on a photomask. The left half of the images is the glass substrate and the right half is the chrome layer. Top: TM-AFM topography image of the surface. Bottom: Image of the same area at the 16th harmonic of the driving frequency. The oscilloscope traces horizontally scan the topography and the 16 th harmonic signals. 
resonance frequencies. Fortunately, the ratio of the third and fundamental resonances did not change significantly as we expected, so we were able to match the resonances in spite of the fabrication variations.

\section{Results and discussion}

We performed our experiments with a commercial AFM system (Dimension 3100, Nanoscope III, Veeco, Santa Barbara, CA) equipped with an external RF lock-in amplifier (SR844). An external lock-in amplifier is used to detect the signals at the 16th harmonic of the driving frequency. Additionally we used a spectrum analyzer to monitor the vibration spectrum of the cantilever.

In Fig. 8 the vibration spectrum of a harmonic cantilever in TM-AFM is given. Apart from the signal at the drive frequency, two peaks (\#6 and \#16) have relatively large signal level compared to their neighbors. These are the harmonics that are closest to the resonance frequencies of the harmonic cantilever. Especially the 16th harmonic has relatively higher signal level compared to its neighbors. That is because the frequency of that particular harmonic matches the third resonance frequency of the harmonic cantilever. In Fig. 9, the spectrum around the 15th-17th harmonics is given when third resonance frequency is exactly equal to 16 times the driving frequency (a) and when there is a slight mismatch (b). This result shows that our correctly-designed cantilevers improve the higher-harmonic signal by 15$20 \mathrm{~dB}$.

In order to demonstrate the sensitivity of imaging at the higher harmonics with harmonic cantilevers, we recorded images of the surface of a photomask with a surface composed of borosilicate glass (modulus of elasticity $=63 \mathrm{GPa}$ ) and chromium (modulus of elasticity $=248 \mathrm{GPa}$ ). In Fig. 10 the TM-AFM topography image (top) and the image at the 16th harmonic (bottom) is depicted together with the oscilloscope traces of the topography and 16th harmonic signal. These figures show that imaging at the 16th harmonic can distinguish between the two materials to a good degree. These images are taken simultaneously and the left side of each image is the glass side and right side is the chromium side.

Since the chromium is stiffer then the borosilicate glass, we expect the contact time to be shorter on chrome side. Fig. 3 suggests that shorter contact time results in larger signals at the 16th harmonic. The oscilloscope traces in Fig. 10 show that the signal at the 16th harmonic is higher on the chrome side as predicted. Additionally, metal traces and/or dust particles on the glass side are quite visible in the 16th harmonic image, demonstrating a better signal-to-noise than in the topographical image. This illustrates a potential additional advantage of higher-harmonic imaging: Surfaces with large topographical differences can be imaged with better resolution than in standard TMAFM.

\section{Conclusion}

The specially micromachined cantilever presented in this report enables tapping-mode higher harmonic imaging, which is proven to be a sensitive technique for measurement of elasticity of surfaces at the nanoscale. Higher harmonic imaging in TM-AFM provides a non-destructive and practical way to map elasticity variations across a surface, and might have advantages for high-resolution imaging of samples with large topography variations.

\section{Acknowledgements}

This work was supported by the birdseed fund of Stanford University Office of Technology Licensing. O. Sahin acknowledges support from a T.J. Rodgers Stanford Graduate Fellowship.

\section{References}

[1] G. Binnig, C.F. Quate, Ch. Gerber, Atomic force microscope, Phys. Rev. Lett. 56 (1986) 930.

[2] Q. Zhong, D. Inniss, K. Kjoller, V.B. Elings, Fractured polymer/silica fiber surface studied by tapping mode atomic force microscopy, Surf. Sci. 280 (1993) L688.

[3] P. Maivald, H.J. Butt, S.A.C. Gould, C.B. Prater, B. Drake, J.A. Gurley, V.B. Elings, P.K. Hansma, Using force modulation to image surface elasticities with the atomic force microscope, Nanotechnology 2 (1991) 103.

[4] M. Heuberger, G. Dietler, L. Schlapbach, Mapping the local Young's modulus by analysis of the elastic deformations occurring in atomic force microscopy, Nanotechnology 5 (1994) 12.

[5] K. Yamanaka, H. Ogiso, O. Kosolov, Ultrasonic force microscopy for nanometer resolution subsurface imaging, Appl. Phys. Lett. 64 (1994) 178.

[6] U. Rabe, K. Janser, W. Arnold, Vibrations of free and surfacecoupl3281ed atomic force microscope cantilevers: Theory Exp. Rev. Sci. Instrum., 67 (1996) 3281.

[7] K. Yamanaka, S. Nakano, Ultrasonic atomic force microscope with overtone excitation of cantilever, Jpn. J. Appl. Phys. 35 (1996) 3787.

[8] O.V. Kosolov, M.R. Castell, C.D. Marsh, G. Andrew, D. Briggs, T.I. Kamins, R.S. Williams, Imaging the elastic nanostructure of $\mathrm{Ge}$ islands by ultrasonic force microscopy, Phys. Rev. Lett. 81 (1998) 1046.

[9] K.B. Crozier, G.G. Yaralioglu, F.L. Degertekin, J.D. Adams, S.C. Minne, C.F. Quate, Thin film characterization by atomic force microscopy at ultrasonic frequencies, Appl. Phys. Lett. 76 (2000) 1950.

[10] M. Stark, R.W. Stark, W.M. Heckl, R. Guckenberger, Spectroscopy of anharmonic cantilever oscillations in tapping-mode atomic-force microscopy, Appl. Phys. Lett. 77 (2000) 3293.

[11] O. Sahin, A. Atalar, Simulation of higher harmonics generation in tapping-mode atomic force microscopy, Appl. Phys. Lett. 79 (2001) 4455.

[12] R. Hillenbrand, M. Stark, R. Guckenberger, Higher-harmonics generation in tapping-mode atomic force microscopy: insights into tip-sample interaction, Appl. Phys. Lett. 76 (2000) 3478.

[13] T.R. Rodriguez, R. Garcia, Tip motion in amplitude modulation (tapping mode) atomic force microscopy: comparison between continuous and point mass models, Appl. Phys. Lett. 80 (2002) 1646.

[14] J. Tamayo, R. Garcia, Deformation, contact time, and phase contrast in scanning force microscopy, Langmuir 12 (1996) 4430. 
[15] A.S. Paulo, R. Garcia, Tip-surface forces, amplitude, and energy dissipation in amplitude-modulation (tapping mode) force microscopy, Phys. Rev. B 64 (2001) 193411.

[16] A.S. Paulo, R. Garcia, Unifying theory of tapping-mode atomic force-microscopy, Phys. Rev. B 66 (2002) 041406(R).

[17] R.W. Stark, W.M. Heckl, Fourier transformed atomic force microscopy: tapping mode atomic force microscopy beyond the Hookian approximation, Surf. Sci. 457 (2000) 219.

\section{Biographies}

O. Sahin received BS degree in electrical engineering form Bilkent University, Ankara, Turkey in 2001 and MS degree in electrical engineering in 2003 from Stanford University, CA. He is currently working towards the $\mathrm{PhD}$ degree in electrical engineering at Stanford University, Stanford, CA. His research interests include developing tools for sensing physical and biological phenomena at the nanoscale.

G. Yaralioglu received his $\mathrm{BS}, \mathrm{MS}$ and $\mathrm{PhD}$ degrees in electrical engineering from Bilkent University, Ankara, Turkey, in 1992, 1994 and 1999, respectively. He is now an Engineering Research Associate at E.L. Ginzton Laboratory, Stanford University, Stanford, CA. His research interests include design, modeling and applications of micromachined ultrasonic transducers and atomic force microscopy at ultrasonic frequencies.

R. Grow received the BA degree in physics from Colorado College, Colorado Springs, in 1996 and is currently working towards the $\mathrm{PhD}$ degree in applied physics at Stanford University, Stanford, CA. His research interests include cantilevers, lithography, MEMS, and nanotechnology.

S.F. Zappe received his Diploma degree in electrical engineering from the Berlin University of Technology, Germany, in 1996. From 1996 until 2001 he worked as a PhD student at the Microsensor and Actuator Center, Berlin University of Technology. In February 2001, S.F. Zappe joined as a postdoctoral researcher to the Stanford Microphotonics Laboratory, Stanford University, CA. His research activities include microfluidic systems for cell and embryo handling, sorting and micro-injection; biology of fruit fly development; gene silencing by means of RNAi (RNA interference); micro-orifices for DNA shearing; micro-fluidic system based re-usable arrays for DNA sequencing; integration of active and passive optical components into microsystems.
A. Atalar received the BS degree from the Middle East Technical University, Ankara, Turkey, in 1974, the MS and PhD degrees from Stanford University, CA, in 1976 and 1978, respectively, all in electrical engineering. In 1979, he was with Hewlett Packard Labs, Palo Alto, CA. From 1980 to 1986 he was on the faculty of Middle East Technical University as an assistant professor. From 1982 to 1983 he was on leave from the University, and was with Ernst Leitz Wetzlar, West Germany, where he was involved in the development of the commercial acoustic microscope. In 1986, he joined the Bilkent University and served as the founding Chairman of the Electrical and Electronics Engineering Department where he is currently a professor. His current research interests include computer-aided design of VLSI and MMIC circuits, micromachined sensors, and actuators. Dr. Atalar is a member of the Turkish Academy of Sciences. He was awarded Science Award of TUBITAK, Turkey in 1994.

C. Quate received his BS from the University of Utah in 1944 and the $\mathrm{PhD}$ degree from Stanford University, Stanford, CA, in 1950. He held positions at Bell Laboratories and Sandia Corporation before joining the faculty at Stanford University, where he has been since 1961. Currently, he is a Faculty Member with the Department of Electrical Engineering and the Department of Applied Physics at Stanford University. His research interests revolve around the scanning probe microscopes. Dr. Quate is a Member of the National Academy of Engineering and the National Academy of Sciences, an Honorary Fellow of the Royal Microscopical Society, and a Foreign Member of the Royal Society London. He has received the IEEE Morris N. Liebmann Award in 1981, the Rank Prize for opto-electronics in 1982, the IEEE Medal of Honor in 1988, the President's National Medal of Science in 1992, and the American Physical Society Keithley Award in 2000.

O. Solgaard received a BS degree in electrical engineering from the Norwegian Institute of Technology, and $\mathrm{MS}$ and $\mathrm{PhD}$ degrees in electrical engineering from Stanford University, California. He held a postdoctoral position at the University of California at Berkeley and an assistant professorship at the University of California at Davis before joining the faculty of the Department of Electrical Engineering at Stanford University in July 1999. His research areas are optical communication and sensing applications of MEMS. He has authored more than 100 technical publications and holds 13 patents. He is a co-founder of Silicon Light Machines, Sunnyvale, California, and an active consultant in the MEMS industry. 